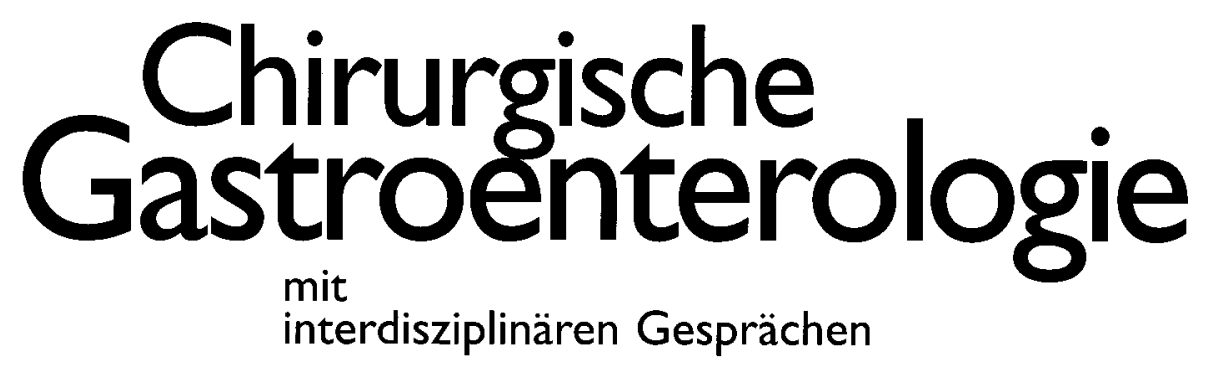

Hauptthema

\title{
H. Friess,
}

Bern

Molekularbiologie

M. von Knebel Doeberitz, Heidelberg (Hrsg.)

\section{in der Viszeralchirurgie}

Eingeladene Beiträge

Interdisziplinäres Gespräch

Weitere Rubriken

Industrieforum

Tagungen und Kongresse 


\section{Band 15, Heft 2, Juni 1999}

\section{Gründungsherausgeber}

A. Akovbiantz $\dagger$, Zürich

H. Denck, Wien

K.-J. Paquet, Bad Kissingen

C. E. Zöckler, Bad Oeynhausen

\section{Hauptschriftleiter}

R. Bittner, Stuttgart

K.-J. Paquet, Hannover

\section{Schriftleitung}

C. E. Broelsch, Essen

M. W. Büchler, Bern

I. Gastinger, Cottbus

K. Glaser, Wien

J. Horn, München

J. R. Izbicki, Hamburg

E. Klar, Heidelberg

J. Mössner, Leipzig

H. Säuberli, Baden

R. Schiessel, Wien

W. G. Zoller, Stuttgart

\section{Wissenschaftlicher Beirat}

J. Alexander-Williams, Birmingham

H. M. Becker, München

M. Betzler, Essen

E. Bodner, Innsbruck

H. Bosseckert, Jena

H. Bülow, Schweinfurt

R. Dölp, Fulda

K. E. Frede, Basel

J. Gallinger, Moskau

A. Gangl, Wien

J. M. Hackl, Innsbruck

E. G. Hahn, Erlangen/Nürnberg

J. Hauss, Leipzig

J. M. Henderson, Cleveland

P. Hermanek, Erlangen/Nürnberg

R. A. Hinder, Omaha

W. Hohenberger, Erlangen/Nürnberg

Y. Idezuki, Tokyo

I. Ihse, Lund

C. W. Imrie, Glasgow

G. G. Jamieson, Adelaide

S. L. Jensen, Århus

K. Junghanns, Ludwigsburg

P. J. Kestens, Brüssel

B. Langer, Toronto

S. Liebe, Rostock

H. Lippert, Magdeburg
P. Malfertheiner, Magdeburg

K. Maruyama, Tokyo

C. Meyer, Straßburg

W. C. Meyers, Worcester

P. Morel, Genf

E. Moreno-Gonzáles, Madrid

V. Pegan, Ljubljana

F. M. Penninckx, Leuven

A. Peracchia, Mailand

R. Porschen, Tübingen

H. B. Reith, Würzburg

J. F. Riemann, Ludwigshafen

L. F. Rikkers, Madison

J. Rodés, Barcelona

H.-D. Saeger, Dresden

R. Schlumpf, Aarau

W.-H. Schmiegel, Bochum

J. Schölmerich, Regensburg

M. Stolte, Bayreuth

K. Sugimachi, Fukuoka

J. Terblanche, Kapstadt

M. Thelen, Mainz

A. Thiede, Würzburg

A. L. Warshaw, Boston

C. Wittekind, Leipzig

Z. Wu, Wuhan

\section{Bibliographische Dienste:}

EMBASE / Excerpta Medica

Reference Update

S. Karger $\cdot$ Medical and Scientific Publishers $\cdot$ Basel $\cdot$ Freiburg $\cdot$ Paris $\cdot$ London $\cdot$ New York $\cdot$ New Delhi $\cdot$ Bangkok $\cdot$ Singapore $\cdot$ Tokyo $\cdot$ Sydney

Die Zeitschrift erscheint dreimonatlich: pro Jahr erscheint 1 Band zu je 4 Heften. Bezugspreis für Jahrgang 15, 1999: DEM 220.- / SFr 172,-- einschließlich MwSt., zuzüglich Postgebühren. Der Abonnementpreis ist im voraus zahlbar. Da Abonnement der Zeitschrift läuft weiter, wenn es nicht spätestens 4 Wochen vor Abschlu $B$ eines Bandes abbestellt wird.

Abonnementbestellungen können bei jeder Buchhandlung oder direkt beim Verlag aufgegeben werden:

S. Karger GmbH

Lörracher Str. 16 a

D-79115 Freiburg

Telefon +49761452070

Telefax +497614520714

Übrige Länder:

S. Karger AG

Allschwilerstr. 10

Postfach

CH-4009 Basel

Telefon +41613061111

E-mail kargergmbh@aol.com Telefax +41613061234

Postbank München 61440-808 E-mail Karger@Karger.ch
Anzeigen: S. Karger

Verlag für Medizin und Naturwissenschaften $\mathrm{GmbH}$

Lörracher Str. 16 a. D-79115 Freiburg, Tel. +49 761452070

Gültig ist die Preisliste Nr. 7 vom 1. Januar 1999

Für den Inhalt außerhalb des redaktionellen Teils (insbesondere Anzeigen, Industrieinformationen, Pressezitate und Kongreßinformationen) übernehmen Schriftleitung, Beirat und Verlag keine Gewähr.

Eine Markenbezeichnung kann warenzeichenrechtlich geschützt sein, auch wenn bei ihrer Verwendung in dieser Zeitschrift das Zeichen ${ }^{\circledR}$ oder ein anderer Hinweis auf etwa bestehende Schutzrechte fehlen sollte. Für Satzfehler, insbesondere bei Dosierungsangaben, wird keine Gewähr übernommen.

Die Zeitschrift sowie alle in ihr enthaltenen einzelnen Beiträge und Abbildungen sind urheberrechtlich geschützt. Jede Verwertung, die nicht ausdrücklich vom Urheberrechtsgesetz zu- gelassen ist, bedarf der vorherigen Zustimmung des Verlags. Das gilt insbesondere für Vervielfältigung, Bearbeitungen, Übersetzungen, Mikroverfilmungen und die Einspeicherung und Verarbeitung in elektronischen Systemen.

(c) Copyright 1999 by S. Karger

Verlag für Medizin und Naturwissenschaften $\mathrm{GmbH}$ Lörracher Straße 16 a, D-79115 Freiburg

Verlagsleitung und presserechtlich verantwortlich: Sibylle Hopf

Redaktionsassistenz: Dr. Hannelore Tenckhoff

Anzeigenleitung: Susanne Meister

Produktionsleitung: Georg Brunner

Herstellung: druckhaus köthen $\mathrm{GmbH}$

Friedrichstraße 11/12, D-06366 Köthen/Anhalt

ISBN 3-8055-6830-4

\section{KARGER}

Fax +49761 4520714

E-mail kargergmbh@aol.com

www.karger.com 
Hauptthema

110 Molekularbiologische Grundbegriffe und Arbeitstechniken Schmid, R. M.; Gansauge, F. (Ulm)

120 Störungen des Zellzyklus und des programmierten Zelltods Ursachen der Tumorentstehung von Knebel Doeberitz, M.; von Knebel Doeberitz, C. (Heidelberg)

128 Tumorprogression: Adhäsion, Proteolyse und Angiogenese

Zöller, M. (Heidelberg/Karlsruhe); Matzku, S. (Karlsruhe/Darmstadt)

138 Molekularpathologie der Ösophagus- und Magenkarzinome Werner, M.; Höfler, H. (München)

144 Hepatozelluläres und cholangiozelluläres Karzinom: Molekularbiologie und Immunpathologie Moehler, M.; Heike, M.; Galle, P. R. (Mainz)

150 Molekulare Veränderungen bei Pankreas- und periampullären Karzinomen

Friess, H.; Kleeff, J. (Bern); Klockow, N.; Ebert, M.; Malfertheiner, P. (Magdeburg); Büchler, M. W. (Bern)

158 Molekularbiologie und Kolonkarzinom: Ein Überblick Wildi, S.; Maurer, C. A.; Büchler, M. W. (Bern)

163 Hereditäre Tumoren des Gastrointestinaltrakts (FAP/ HNPCC)

Gebert, J.; von Knebel Doeberitz, C. (Heidelberg)

173 Bedeutung disseminierter Tumorzellen bei gastrointestinalen Tumoren

Weitz, J.; Koch, M.; von Knebel Doeberitz, M. (Heidelberg)

181 Gentherapie bei gastrointestinalen Tumoren am Beispiel des Pankreaskarzinoms: Standortbestimmung und Perspektiven

Kleeff, J.; Friess, H. (Bern); Korc, M. (Irvine); Büchler, M. W. (Bern)

188 Immuntherapie bei gastrointestinalen Tumoren Zöller, M. (Heidelberg/Karlsruhe); Matzku, S. (Karlsruhe/Darmstadt)
Main Theme

110 Molecular Biological Basic Principles and Techniques Schmid, R. M.; Gansauge, F. (Ulm)

120 Disturbances of the Cell Cycle and Apoptosis - Causes of Tumor Development von Knebel Doeberitz, M.; von Knebel Doeberitz, C. (Heidelberg)

128 Tumor Progression: Adhesion, Protheolysis, and Angiogenesis

Zöller, M. (Heidelberg/Karlsruhe); Matzku, S. (Karlsruhe/Darmstadt)

138 Molecular Pathology of Esophageal and Stomach Carcinomas Werner, M.; Höfler, H. (München)

144 The Hepatocellular and Cholangiocellular Carcinomas: Molecular Biology and Immunopathology Moehler, M.; Heike, M.; Galle, P. R. (Mainz)

150 Molecular Alterations in Pancreatic and Periampullary Cancers

Friess, H.; Kleeff, J. (Bern); Klockow, N.; Ebert, M.; Malfertheiner, P. (Magdeburg); Büchler, M. W. (Bern)

158 Molecular Biology and Colorectal Cancer: An Overview Wildi, S.; Maurer, C. A.; Büchler, M. W. (Bern)

163 Hereditary Tumors of the Gastrointestinal Tract (FAP/ HNPCC)

Gebert, J.; von Knebel Doeberitz, C. (Heidelberg)

173 Importance of Disseminated Tumor Cells in Gastrointestinal Tumors

Weitz, J.; Koch, M.; von Knebel Doeberitz, M. (Heidelberg)

181 Gene Therapy in Patients with Gastrointestinal Tumors Taking Pancreatic Carcinomas as an Exemple: State of the Art and Perspectives Kleeff, J.; Friess, H. (Bern); Korc, M. (Irvine); Büchler, M. W. (Bern)

188 Immunotherapy in Gastrointestinal Tumors Zöller, M. (Heidelberg/Karlsruhe); Matzku, S. (Karlsruhe/Darmstadt)

\section{KARGER}

Fax +497614520714

E-mail kargergmbh@aol.com

www.karger.com
(C) 1999 S. Karger GmbH, Freiburg

Artikel (Volltext) und Inhaltsverzeichnisse sowie das vorläufige Inhaltsverzeichnis des nächsten Heftes: www.karger.com/journals/cga/cga_bk.htm 
Interdisziplinäres Gespräch

195 Molekularbiologie in der Viszeralchirurgie - prädiktive Diagnostik hereditärer Tumoren

Schackert, H.-K. (Dresden) (Gesprächsleiter)

\section{Industrieforum}

204 Tagungen und Kongresse

106 Impressum

U2 Hinweise für Autoren (2. Umschlagseite)
Interdisciplinary Discussion

195 Molecular Biology in the Visceral Surgery - Predictive Diagnostics of Hereditary Tumors

Schackert, H.-K. (Dresden) (Discussion Leader)

202 Industrial Forum

204 Meetings and Conferences

106 Imprint

U3 Guidelines for Authors (Inside back cover)

\section{In Vorbereitung}

Interdisziplinäres Gespräch: Neoadjuvante und adjuvante Therapie beim Ösophaguskarzinom

Petrasch, S. (Bottrop)

Antibiotikaprophylaxe in der Gallenwegchirurgie Wittmann, D. H. (Wisconsin)

Hepatikolithiasis - endoskopisch-interventionelle Therapiemöglichkeiten Jakobs, R.; Riemann, J. F. (Ludwigshafen)

Primär sklerosierende Cholangitis - Therapieoptionen

Maier, K. P. (Tübingen)

Indikation und Ergebnisse der Lebertransplantation bei primär sklerosierender Cholangitis Otto, G. (Mainz)

Leiomyosarkom der Leber und Vena-cava-Kompressionssyndrom bei einer 66jährigen Patienten - eine Kasuistik

Kahlenberg, D.; Will, U.; Scheele, J.; Werner, B.; Katenkamp, D.; Bosseckert, H. (Jena)

Subklinische portosystemische Enzephalopathie und Lungenstrombahnveränderungen bei Kindern und Jugendlichen mit portaler Hypertension bei prähepatischem Block Otting, U.; Hellmann, C.; Planke, C.; Lehmann, R.; Gottschalk, S. (Berlin)

\section{KARGER}

Fax +497614520714

E-mail kargergmbh@aol.com

www.karger.com 